\title{
versants
}

\section{La «storia di tutti» secondo Pennacchi (con una breve nota sugli anni Sessanta)}

Georgia FIORONI

\begin{abstract}
Al di là del notevole impianto narrativo, Canale Mussolini. Parte seconda [20I5] di Antonio Pennacchi ha il merito di raccontare un periodo di storia italiana attraverso una «voce collettiva»: è la voce del dialetto, di cui qui si intende studiare la portata. Ma il valore della scelta linguistica dipende anche dall'emigrazione cui sono costretti i personaggi della saga di Pennacchi (iniziata con Canale Mussolini [20I0]): un'epopea, la loro, che termina all'inizio degli anni Sessanta. E un'emigrazione diversa, ma per certi aspetti non meno violenta, tocca anche alcuni romanzi editi proprio negli anni del boom economico, ai quali qui (in forma di aggancio) si dedicherà qualche osservazione conclusiva.
\end{abstract}

Keywords: romanzo, letteratura moderna e contemporanea, Antonio Pennacchi, Natalia Ginzburg, Luciano Bianciardi, Paolo Volponi, Lucio Mastronardi.

Quel romanzo parla della storia di tutti. È la voce collettiva. Io l'ho solo messa in pagina e fatta cantare.

Antonio Pennacchi

\section{Premessa}

Se è vero che la lettura di un paese o di un periodo storico passa anche attraverso la lettura della sua produzione letteraria e delle sue peculiarità linguistiche, è ancora più vero che molto spesso tale lettura sembra rivelarsi più efficace in testi che si snodano attorno all'emigrazione.

Un esempio significativo in tal senso è una pubblicazione recente, Canale Mussolini. Parte seconda (2015) di Antonio Pennacchi: di questo romanzo (continuazione della saga aperta da Canale Mussolini [20Io], scoperta che devo all'amico e maestro Giovanni Bardazzi) si intende sottolineare l'impiego del dialetto, concludendo con qualche osservazione di aggancio su altri romanzi siglati da un trasferimento, editi e ambientati proprio nel periodo su cui si chiude l'«epopea» di Pennacchi, vale a dire l'inizio degli anni Sessanta. In effetti, se Canale Mussolini (vincitore del Premio Strega nel 20Io) racconta attraverso le vicende della famiglia Peruzzi (che dalla pianura padana si trasferisce nelle Paludi Pontine) mezzo secolo di storia italiana, la Parte seconda 
prosegue la «saga» ${ }^{1}$ (non esente da toni onirico-fantastici ${ }^{2}$ ), prendendo avvio dal 25 maggio 1944 (data della fine della guerra a Littoria, oggi Latina), per giungere all'Italia degli anni Sessanta, attorno a cui si snodano opere quali Il calzolaio di Vigevano [1959/1962] di Lucio Mastronardi, La vita agra [1962] di Luciano Bianciardi e Memoriale [1962] di Paolo Volponi.

\section{Verso Sud: Canale Mussolini. Parte seconda}

Canale Mussolini racconta la discesa dei Peruzzi (e di tutto un universo di personaggi che a loro sono legati) dal Nord-Est italiano, verso Sud. Tale discesa, dettata dalla prospettiva di un lavoro (la bonifica delle Paludi Pontine), è ampiamente descritta in termini di «esodo» nel II capitolo della prima parte della saga:

Fu un esodo. Trentamila persone nello spazio di tre anni - diecimila all'anno - venimmo portati quaggiù dal Nord. Dal Veneto, dal Friuli, dal Ferrarese. Portati alla ventura in mezzo a gente straniera che parlava un'altra lingua. Ci chiamavano «polentoni» o peggio ancora «cispadani». Ci guardavano storto. E pregavano Dio che ci facesse fuori la malaria. | Fu un esodo [...]. [...] Fu un esodo però, le ho detto. | In trentamila in quasi tre anni ci caricarono sui treni e ci portarono qui. Sulle tradotte. A scaglioni. Un treno al giorno. Diecimila all'anno. Facendoci attraversare tutta Italia (Pennacchi 2010: 137 e I48).

È un esodo che accomuna migliaia di lavoratori del Nord che si spostano per costrizione («È lì che ha avuto inizio la nostra storia; costretti ad emigrare in Agro Pontino - nudi come vermi - per ricominciare da capo tutta la generazione nostra dei Peruzzi e quelle che verranno», 20I0: 135) e per fame («Per la fame. Siamo venuti giù per la fame. E perché se no? Se non era per la fame restavamo là. Quello era il paese nostro», 20I0: 9).

\section{«Gente straniera che parlava un'altra lingua»}

«Per la fame» e «nudi come vermi», migliaia di persone - perlopiù analfabete («[...] e qualche donna che sapeva leggere, guardando le insegne dal

\footnotetext{
I «Epopea», «saga» e «gesta»: così, in più occasioni (quarta di copertina, interviste, ecc.), sono stati definiti i due romanzi di Antonio Pennacchi.

2 In un'intervista di Matteo Nucci, Pennacchi, sollecitato sulla sua idea di «fantastico» e «magico», risponde: «Io ho amato molto Jorge Amado ma sono un marxista materialista e il mio magico-religioso viene dal mondo contadino. Non è realismo magico. Semmai iper realismo magico. Cioè, io racconto quel che si racconta qui. Del fantasma di Mussolini che gira in moto per queste strade si sa da sempre. E io parlo al mio popolo, alla mia terra, ai miei compagni di strada, a Latina» (Pennacchi 20I5b).
} 
finestrino aveva detto: "Roma!" [...]», 2010: 153) - improvvisamente si trovano in mezzo a «gente straniera che parlava un'altra lingua» (2010: 137). Da quei luoghi quasi nessuno si muoverà più; ma il legame sottile con la terra natale sarà garantito da una fedeltà alla lingua delle origini, seppur contaminata da influenze laziali.

La centralità dell'aspetto linguistico del romanzo è confermata nella Nota filologica dell'autore:

Il dialetto veneto-pontino che si parla in Canale Mussolini non è più, naturalmente, quello di Goldoni né - tanto meno - quello che si parla in Veneto oggi. Quando, per esempio, ci incontriamo con le mie cugine che sono rimaste lassù, qualche volta facciamo fatica a capirci. Il nostro è un impasto di rovigotto, ferrarese, trevigiano, friulano eccetera - contaminato da influenze laziali - privo di strutturazione grammaticale fissa, con le vocali ora aperte ora chiuse e le desinenze che cambiano da podere a podere e da situazione a situazione, anche spesso nello stesso parlante. Questo è però l'impasto che ho imparato da mia madre, e che probabilmente ho contaminato a mia volta nel corso degli anni (2010: 457).

Operazione analoga prosegue (lo ribadisce un'altra Nota) nel secondo volume: «L'impasto linguistico dei dialoghi [...] rimane l'arcaico veneto-ferrarese della bassa rovigotta - contaminato probabilmente in Agro Pontino - appreso bambino da mia madre e dai miei zii, e definibile oggi come dialetto veneto dell'emigrazione» (Pennacchi 2015a: 427) . Tuttavia, accanto all'impasto veneto-pontino, al lettore può accadere di imbattersi anche in altri dialetti, che, di volta in volta, assumono varie funzioni. In alcune situazioni, semplicemente, calano il lettore in realtà geografiche diverse da quelle della zona di Latina: è il caso, ad esempio, del periodo napoletano di zia Santapace e del marito Benassi (che, a sua volta, ha un'inflessione um$\left.\mathrm{bra}^{4}\right)$, nel corso del quale una donna napoletana vittima della gelosia di zia Pace si difende così: "I nun l'aggio mai tuccato, nun date retta a 'e cchiacchiere signo', che la gente è cattiva e calunniosa" e mia zia invece gridando insisteva sempre più, con le mani sempre al collo» (2015a: I29). Altrove, invece, la scelta del dialetto costituisce un documento storico: così avviene nel racconto - in dialetto romanesco e graficamente segnalato dal corsivo, giacché tratto da un ricordo reale ${ }^{5}$ della battaglia di Porta San Paolo da parte

3 La nota prosegue con la seguente precisazione: «Ho trovato però utili, seppure di area padovana: S. Belloni, Grammatica veneta, Padova 2009; L. Nardo, Dizionario italiano-veneto. A sercar parole, Padova 2009".

4 Si legga l'esempio seguente: «[...] strillava zio Benassi: "Ma io ho fatto Littoria con le mani mie, ce so' partito dall'Umbria in bicicletta pe' venilla a fa', e tu adesso me vòi riportà in mezzo alla fanga?" [...]» (Pennacchi 20I5a:36I).

5 Scrive Pennacchi nella sezione Bibliografia essenziale: «Il racconto del ragazzino a Porta San 
di un ragazzino che, all'epoca dei fatti, aveva dieci anni: «Er ricordo più vivo che ciò» [...] "so' i militari italiani sdraiati pe' tera che sparaveno addosso ai tedeschi che je staveno de faccia. Se facevano scudo puro dietro a de li tranve arivortati. Li sordati, sia italiani che tedeschi, cascaveno come pupazzi e rimaneveno lì stecchiti» (20I5a: I6I). In altre occorrenze il dialetto caratterizza - quasi folcloristicamente - una tipologia regionale: ad esempio nella rabbia espressa da alcuni toscani del $2 \mathrm{I}^{\circ}$ reggimento fanteria Cremona nei confronti del principe Umberto: «Un toscano urlò: "La horda al hollo, ai Savoia!" | Un altro: "O finohhio, màndaci il tu babbo"» (2015a: 245). Oppure ancora ricalca la lingua di personaggi pubblici identificabili con nome e cognome: così avviene per il siciliano Mario Scelba: «Scelba aveva la fobia del comunismo: "Chisti annu 'u pianu Kappu”, diceva ogni tanto a De Gasperi, “chisti s'arrivòtanu e po' cumànnanu iddi”, e giù col manganello - ma non solo, purtroppo, il manganello - della Celere, che aveva peraltro inventata lui» (2015a: 383).

Al di là di queste escursioni, a predominare nettamente è il «dialetto veneto», ma - si badi bene - il dialetto «veneto» dei protagonisti che abitano le campagne circostanti Littoria, poiché, almeno nei fatti, chi si è insediato nella cittadina tenta di mimetizzarsi - linguisticamente - con i locali:

E mentre noi in campagna continuavamo a parlare in veneto, a Littoria - con tutti questi romani e soprattutto con i loro figli - da allora e per sempre s'è parlato romanesco; pure i laziali marocchini delle montagne qui intorno e gli operai cispadani altitaliani polentoni delle Case popolari, che non volevano sfigurare davanti ai romani della piazza, i piazzaroli (2015a: I00).

Il veneto - ma questo vale anche per gli altri dialetti - assurge a vera e propria lingua: al pari dell'italiano, dell'inglese, dell'americano e di altri dialetti. Diomede - il grande personaggio della seconda parte di Canale Mussolini - parla l'americano e il tedesco «come il veneto di casa sua», conosce l'inglese, ma l'italiano «non tanto ancora»: «S'era imparato pure l'inglese. L'italiano non tanto ancora, ma il tedesco e l'americano oramai li parlava come il veneto di casa sua» (Pennacchi 20I5a: 277). Lo avrà sicuramente imparato alla fine della guerra, nel I95I, quando per la prima volta si reca a Milano: «Diomede era la prima volta - poverino - che veniva a Milano, che ne sapeva dei dialetti loro? Lui conosceva il veneto, l'italiano, il romanoumbro-marocchino, il tedesco e l'americano ancora ancora. Ma il milanese no» (20I5a: 404). È il milanese della sua futura moglie, sapientemente e ironicamente inserito nel testo ${ }^{6}$ proprio per ribadire, ancora una volta, l'idea

Paolo: Alvaro Cardarelli, in "Rione Garbatella", http : // www.rionegarbatella.it/archivio-racconti-garbati/486-a-difesa-de-roma-ricordi-personali-dell8-e-9-settembre-1943» (2015a: 429). 6 «A un certo punto però Diomede apre la porta del bagno e ne esce tutto nudo bagnato con le sue grazie-disgrazie di fuori - con le braccia e le mani alte, ad asciugarsi la testa. | Lei 
che lingue ufficiali, parlate regionali e dialetto stiano esattamente sullo stesso piano.

\section{Dialet to senza confini}

Se l'impiego del dialetto qui delineato sembra ricalcare tendenze più volte appurate dalla critica - ad esempio una resa più naturale dei dialoghi e una più fedele caratterizzazione dei personaggi e della loro provenienza -, appare invece straniante il suo dilagare quasi iperbolico: il fatto che un numero impressionante e variegato di personaggi - di regioni italiane diverse e, addirittura, di altre nazioni - si esprima, pensi, si arrabbi, si sorprenda e si emozioni in veneto-pontino, genera quasi un effetto di spaesamento nel lettore. La ragione di tale scelta non resta senza risposta, ma giunge all'inizio di Canale Mussolini, quando il narratore reagisce con stizza alle critiche del suo anonimo interlocutore, che si beffa di un Mussolini in versione veneta:

Comunque finito il comizio - quello nostro del I9II e della Libia - sceso dal palco, la gente gli si è fatta intorno a salutarlo e coi più stretti sono andati a bere un bicchiere come si fa di solito. Pure mio nonno ha fatto il gesto di avvicinarsi per salutarlo, anche se era un po' intimidito perché pensava che non si ricordasse. Invece come lo ha visto, il Mussolini ha strillato: «Peruzzi! Am dispiase propi ma stavolta n'an pòi vegner a magnar da valtri, ch'agò d'andar via. Ma nol mancarà ocasion, t’al sicuro». | E perché ride adesso? Cosa dice? Lei dice che non può essere che Mussolini parlasse così, perché lui era romagnolo di Predappio, tutto un altro dialetto, un'altra inflessione? | Lei la deve smettere con queste fesserie, io mica sto qui a raccontare barzellette. Cosa vuole che ne sappia io di quale dialetto e con quale inflessione parlasse Mussolini? Quelle sono però le cose che ha detto - la sostanza - e io gliele ridico parola per parola esattamente nello stesso dialetto in cui le hanno dette a me. Io non cambio niente. [...] Ciò che conta è la tradizione, la lingua che parla colui che racconta, e a me me l'hanno raccontata così e io così la riracconto a lei (2010: 43-44).

«Ciò che conta», dunque, «è la tradizione», la «sostanza»: le gesta dei Peruzzi e di una parte del paese sono state tramandate in questo dialetto, e in questo dialetto il narratore le propone al lettore. Alternative non ce ne sono, se non a costo di tradire la storia attraverso una lingua e un'inflessione che con quella saga poco o nulla hanno a che fare, poiché quella saga vive e si nutre dell'impasto linguistico dei protagonisti che l'hanno creata, subita e, in alcuni casi, anche amata.

chinata dall'altra parte sul letto, alza lo sguardo. Lo vede e sbarra gli occhi : "Ostia che bìgol!" incredula» (2015a: 404). 
Vale la pena, per rendersi conto concretamente della portata del dialetto nei due libri di Pennacchi, osservare più da vicino chi, e in che misura, si lascia contaminare da questa parlata che, pur attraversando già la prima parte dell'epopea, coinvolge un numero e una tipologia notevole di individui anche nella Parte seconda. A esprimersi in veneto-pontino sono personaggi pubblici italiani del calibro di Badoglio ("Il Cln?” rispondeva Badoglio: "El governo son mi, e comando su valtri anca mi"”, 2015a: 196), di Togliatti («[...] quando finalmente dall'URSS è sbarcato a Napoli Togliatti e due giorni dopo, il 29, s'è presentato davanti al consiglio nazionale del suo partito: "Fermi tutti! Qui se ga da ripensar qualcossa"», 2015a: 196), del re Vittorio Emanuele III di Savoia («Sìo mati? Il re son mil Come casso av permetì?», 20I5a: 196) e, ancora una volta, del duce, durante il discorso pubblico pronunciato nel settembre 1943 da Monaco, via radio, all'Italia:

«E vinseremo!» ha detto il Duce, e uscito di là è tornato a Monaco da Pavolini e Farinacci, il I6 settembre alla radio ha fatto comunicare all'Italia dall'etere tedesco, la sua nuova, buona, ultima novella: «Popolo italiano non demordere, mi son tornà. Fasso 'a repubblica e con tuti valtri di nuovo ale armi con $\mathrm{mi}$, marseremo insieme fino ala vitoria al fianco de l'aleato zermànico da senpre! Contenti?» (2015a: 177).

Ma Pennacchi va oltre: il suo dialetto non conosce i confini nazionali e diventa lingua di comunicazione fra Hitler e Mussolini («Agò le armi segrete, Benito! Agò questo, agò st'altro» 20I5a: 177$)^{7} \mathrm{e}$ fra Stalin e Togliatti («E Stalin subito: "Comandi, sior! Sarà fatto”» 2015a: 203) ${ }^{8}$. Anche Churchill si presenta in versione veneta («Era stato Churchill il primo a dire: "Femo una cortina de fero par isolar quei demoni dell'Urss: vade retro, Satana! N'i pasaran!"», 2015a: 38I) e a questa operazione linguistica non si sottraggono nemmeno le conversazioni fra De Gasperi e Truman: "Ma figùrete, Alside..." gli rispose Truman, presidente degli Usa: "Tuti i schei che ti vol! Basta ch'at mandi via i comunisti. Se no gninte". | "Cossa agò da fare mi?", disse De Gasperi a Togliatti» (2015a:382).

7 Altro esempio significativo nel passo seguente: «[...] "Grassie tante Adolfo d'averne restituì sano e salvo a me fameja, ti te sì propri un grand'amiso. Ma desso va in pace lasia 'ndare in pace anca mi". | Ma niente da fare. Quello lo aveva di nuovo rintortolato come un calzino: "Agò le armi segrete, Benito! agò questo, agò st'altro. Con ti al mi fianco par senpre, non podarem che vìnsere e vìnsere"» (2015a: 177).

8 «Ma lei pensi che c'è perfino qualche storico che dice che l'idea non sarebbe stata neanche di Stalin, ma addirittura di un ambasciatore del re e di Badoglio che avrebbe convinto Vysinkij: "Dighe a Stalin che ciama 'l Toliatti e ghe dise: così e cosà". E Stalin subito: "Comandi, sior! Sarà fatto"» (2015a: 203). 
Il fenomeno non si limita a coinvolgere personaggi precisi, ma anche intere comunità, come i componenti dell'esercito tedesco («Il merito però con Hitler se lo prese tutto Otto Skorzeny - un capitano SS aggregato a loro - un gigante di oltre due metri presente in ogni fotografia: "Agò fato tuto mi, vardè qua!" disse al Führer al ritorno", 20I5a: 176) e gli alleati, che si esprimono attraverso un particolare impasto linguistico che talvolta prevede l'inserzione dell'inglese in brevi frasi presentate in italiano nel testo, ma in realtà anch'esse pronunciate in inglese («[...] questi neri americani» [...] pigliavano in giro i loro commilitoni bianchi: "Guarda qua!" in inglese. "Tu invece short! Short”» 20I5a: $\left.280^{9}\right)$, talaltra lascia che sia il dialetto a prevalere:

E quando questi hanno visto - dentro la sede loro - tutto quel casino là fuori, si sono detti anche loro: «Porca putana, cossa l'è drìo susèdere? Ndemo a védare, ciò!» e si sono avviati. [...] Ma neanche chinato e guardato, subito uno dei tre s'è rialzato a dire di scatto agli altri due - ma piano piano, solo a loro due, perché nessun altro sentisse - «Porca putana, fradèi! Ma questo xè Big Boss, Big White Boss!» (2015a: 285).

Un impiego di questo tipo, oltre a generare effetti di comicità straniante, accentua la dimensione orale del racconto - la volontà di enfatizzare il tramandarsi di una vicenda - di cui ora si vuole $\left(\mathrm{si} \mathrm{deve}^{\mathrm{io}}\right)$ lasciare una traccia scritta. Ma tale strategia ha una funzione di livellamento: i personaggi che hanno fatto la storia - tutti i personaggi che hanno fatto la storia, siano essi pubblici e conosciuti oppure privati e anonimi - sono posti sullo stesso piano. L'impasto linguistico adottato stempera (e a tratti annulla) il confine tra storia pubblica e storia privata, affinché l'entrata della grande Storia nella storia di una famiglia venga percepita in tutta la sua portata: a ben pensarci un'operazione simile - benché con modalità e risultati molto diversi - era stata svolta da Natalia Ginzburg nel suo Lessico famigliare ${ }^{\text {II }}$. Pertanto le os-

9 Procedimento analogo sembra verificarsi nelle parole dell'autista ispano-americano: «L'autista ispano-americano quel giorno [...] non volle nemmeno che gli pagassero la corsa: "Omaggio! È stato un onore Big Boss. Yo soi encantado d'hacer vuestro conocimiento". Ma prima d'andarsene si riavvicinò di nuovo a Diomede e piano piano, bisbigliando, gli chiese: "Ma no se poderìa ridarghe un'ociata? Mi no go visto gninte là, mi stevo par de soto"» (Pennacchi 2015a: 290-9I), ove è verosimile immaginare che le parole riportate in italiano siano invece state pronunciate in inglese.

Io Si leggano le parole di Pennacchi in un'intervista di Mattia Nucci del 20I5: «Io l'ho detto e lo ripeto: sono venuto al mondo per raccontare questa storia qui. Quando ho letto $\mathrm{Il}$ mulino del Po di Bacchelli o Placido Don di Solochov mi era già chiaro che avrei voluto scrivere un secolo di storia attraverso le vicende della mia famiglia» (Pennacchi 20I5b).

II Tornano utili le parole (attribuite a Italo Calvino, seppur senza firma) riportate nel risvolto editoriale della prima edizione di Lessico famigliare: «[...] La Ginzburg ha voluto evitare ogni invenzione come ogni indeterminatezza: i personaggi vi sono designati col nome e cognome della loro vera identità; e se si facesse un "indice dei nomi" del libro vi si vedrebbero allineate molte delle figure più famose della vita politica, sociale, letteraria, universitaria, con lo stesso 
servazioni di Enrico Testa sulla scrittrice sembrano (almeno in parte) valere anche per Pennacchi, poiché la sua lingua (analogamente all'«italiano medio e parlato» della Ginzburg) sembra «destinat[a] all'espressione non dei soliti contenuti realistici, ma, ad un primo livello, alla messa in scena di una conversazione che resta» e, «ad un livello più profondo, [...] è [...] abilitata a svolgere un'operazione dalla complessa trama antropologica: un rituale funerario di ricongiungimento con le amate voci perdute nell'aldilà coincidente con la ricostituzione dell'idioletto della propria tribù famigliare»(Testa 1997). Tale «ricongiungimento» sembra verificarsi, se si dà fede alle parole di Pennacchi, anche in Canale Mussolini (e, di conseguenza, anche nella Parte seconda): «Canale Mussolini in parte l'ho scritto sotto dettatura dei miei morti. Di mio padre e mia madre, della gente che ho conosciuto e che non c'è più. Loro sono come i penati dell'antichità. Le loro voci mi arrivano dentro e a volte mi fanno piangere» (Pennacchi 20II).

Tuttavia, il fatto che l'autore non ricorra a un italiano medio, ma impieghi in maniera quasi iperbolica il dialetto che ha sentito e ha imparato, potrebbe condurre a una dimensione di innaturalezza, se non fosse che tale procedimento è asistematico: fra i tanti esempi, si pensi alle parole pronunciate da Togliatti («[...] quando finalmente dall’URSS è sbarcato a Napoli Togliatti e due giorni dopo, il 29, s'è presentato davanti al consiglio nazionale del suo partito: "Fermi tutti! Qui se ga da ripensar qualcossa"», Pennacchi 20I5a: I96), riprese e variate, poco oltre, in italiano: «[...] il 29, ha riunito il consiglio nazionale e ha detto: "Fermi tutti, compagni, contrordine! Prima si libera insieme agli altri l'Italia dal nazismo e dal fascismo. [...]"» (2015a: 200). Si registrano poi casi in cui il personaggio resta tendenzialmente fedele alla sua lingua: il soldato Eberahrd (ed è forse l'esempio più incisivo), pur parlando «bene l'italiano - anzi, quasi quasi parlava meglio il veneto» (20I5a: 27), tende ad esprimersi in tedesco. Tale scelta non è casuale, dal momento che Eberarhdt è legato a Diomede da un sodalizio di amicizia e di amore omoerotico (forse il più profondo legame di tutto il libro). Non tradire l'amico, nemmeno nella rimembranza, significa non tradirlo nemmeno linguisticamente: per questo motivo le parole del soldato restano impresse in tedesco (persino nei gesti!) anche nel ricordo onirico di Diomede: "Quello [Eberhardt, nel sogno] s'è voltato - sempre coi fogli arrotolati sotto il braccio - e con la testa faceva segno di no: "Nein, nein, nein", ma solo a gesti però, non con le parole» (20I5a: 378 ); e in tedesco (nella sua lingua) - non in italiano, né in dialetto - sono le ultime parole pronunciate dal soldato prima di morire, quando Diomede tenta inutilmente di rianimarlo: "Ich liebe dich, Diomoedes” e poi è morto» (20I5a: 8I). Ma il loro connubio si ri-

rilievo dei più oscuri parenti e conoscenti, nella prospettiva che loro tocca non nella Storia, ma nelle nostre storie private». 
versa anche sul piano musicale, un tenore-primo (quasi soprano) Eberhardt e un tenore-secondo (quasi baritono) Diomede: ad esempio nell'esibizione comune del Salve Regina (che il primo insegna al secondo «perfetta perfetta in latino e in gregoriano»), che Diomede non manca di cantare «da solo in bicicletta», ma sempre con l'impronta dell'amico dal momento che, anche in solitudine, «nella pronuncia delle parole», «c'era un qualcosa di strano, un qualcosa che non tornava. Più ancora che veneto-latina aveva - come dire? - un'inflessione teutonico-germanica» (2015a: 33). Il connubio musicale accompagna Diomede anche nel momento di lasciare definitivamente l'amico morto, «con dentro la testa la voce di Luciana Dolliver che frullava, come dalla radio: "Non partir, non partir!"» (2015a: 83).

\section{Tra parlato e scritto}

L'elemento musicale sposta gradualmente il discorso dalla dimensione orale a quella scritta. Se il parlato è segnalato semplicemente dalle virgolette (senza distinzione grafica fra dialetto, italiano e altre lingue), ciò che attiene alla traccia scritta viene trattato diversamente.

Questa procedura non riguarda solo casi di parole scritte su una pagina, ma anche esempi che si muovono in una zona di confine: lo confermano i motivi musicali che percorrono la Seconda parte di Canale Mussolini e risaltano per disposizione grafica particolare (generalmente posti al centro della pagina, in corsivo, aperti e chiusi dalle virgolette, e talvolta trascritti per intero). Il trattamento non segue distinzioni: vale per le canzoni fasciste (l'Inno del balilla, Pennacchi 20I5a: I8); per le canzoni di lotta, siano esse cantate in italiano (La canzone di Paralup, 20I5a: 22I) o modificate con inserzioni dialettali (Campane di Monte Nevoso, 20I5a: 376); per i canti popolari (Sul ponte di Bassano [20I5a: 253], qui lievemente dialettizzata e con invenzione del finale); per l'opera (è il caso dell'Aida, di cui si riproduce la romanza Celeste Aida [20I5a: I25] e il duetto finale $O$ terra, addio [20I5a: I25-26]); per le cantilene improvvisate (Batosin d'un Batoseto, 2015a: 293); per il latino e natalizio Adeste fideles (20I5: 333), come pure per le canzoni in voga in quegli anni (la già citata Non partir [20I5a: 83], Zingara [2015a: 297-98], Gli zingari [2015a: 298-99], La luna nel rio [2015a: 367], Reginella [2015a: 34I]), talvolta trasformate in dialetto (è il caso di Ti voglio tanto bene di Claudio Villa [20I5a:313]).

Un discorso analogo, che qui ci si limita a segnalare con qualche (non esaustivo) esempio in nota ${ }^{12}$, riguarda anche un'altra sfera - ancora una vol-

I2 «Era il 25 maggio 1944, giovedì, san Beda e sant'Urbano - "Per sant'Urbano il frumento è fatto grano" - liberazione di Latina» (2015a: I0); «E rinacque a nuova vita come un fungo dopo la pioggia quella mattina di lunedì 29 maggio 1944, san Massimo vescovo - "Tra maggio e giugno I fa il bono fungo" [...]» (2015a: I44); "Accerchiarono la zona e alle cinque e mezzo del mattino - oramai 22 marzo 1944, martedì, san Benedetto: "La rondine è sotto il tetto" - raggiunsero le misere capanne in cui quelli tranquilli dormivano, e gli intimarono: "Arrendetevi!"» (2015a: 
ta scritta ma connessa all'oralità - ossia il repertorio e la saggezza popolare costituiti dai proverbi che percorrono il romanzo con una certa regolarità a meglio scandire il trascorrere del tempo e a sottolineare date importanti (tanto sul piano storico tanto sul piano individuale).

In ambito strettamente scritto - oltre alle regole grafiche appena menzionate - si osserverà la tendenza all'italiano, sia nella sfera privata sia in quella pubblica: Mussolini parla in veneto, ma nella realtà più intima (ora documentata) - nella realtà che i protagonisti della saga di Pennacchi non hanno potuto (all'epoca) accertare -, vale a dire nelle lettere indirizzate a Clara Petacci, scrive in italiano:

Nessuna legittimità e nessuna giurisdizione vera. Su territori e forze armate, chi comandava erano i tedeschi e neanche due mesi dopo avere proclamato la Repubblica sociale italiana Rsi - o repubblica di Salò - è Mussolini stesso a scrivere a Claretta il 20 novembre 1943: «La mia giornata è sempre più dura e arida. Vivo solo. Non parlo con nessuno. Mi sento circondato. Non mi si vuole dare la possibilità di muovermi. Quando mi muovo, l'apparato italo-germanico di protezione è imponente. Io taccio» (2015a: 178$)^{13}$.

Fa eccezione, ma non poteva essere diversamente, zia One, che parla e scrive in dialetto ( $\mathrm{E}$ a lei - zia One - era cresciuta [la nostalgia] di loro, e scriveva alla mamma, mia nonna, di nascosto dal marito: "Ditte al fratelo Pericle cal trova lu, bona sistemasion pel me omo"», Pennacchi 2015a: I03), naturalmente perché in quella realtà il confine fra scritto e parlato (la coscienza del confine fra scritto e parlato), quasi, non esiste.

Tende poi a una sorta di standardizzazione e di uniformità rispetto al contesto in cui è collocato, tutto ciò che ha a che fare con la sfera collettiva, in un intento di autenticità documentabile: dall'epitaffio sulla tomba «al cimitero vecchio di Latina» (20I5a: 47-48), al «foglietto» appeso al portone della chiesa di San Marco (20I5a: 48), passando per le ragioni dell'attribuzione della medaglia di bronzo al maresciallo del carabiniere Luigi Zaccheo, detto «Fiorello» («La motivazione della sua medaglia di bronzo recita: “Coraggioso patriota, subito dopo l'armistizio si prodigava senza sosta nell'organizzare un nucleo di patrioti [...] rivelando, in ogni circostanza, eccezionali doti di animatore ed elevato spirito combattivo”», 20I5a: 63 $3^{14}$ ), per gli scritti di De Gasperi («E l'II febbraio

190); «Diomede compì ventun anni e divenne finalmente maggiorenne il I2 maggio I947 - lunedì, santa Rossana: "D'aprile piove per gli uomini, / maggio invece per le bestie" - il giorno che De Gasperi cacciò comunisti e socialisti dal governo» (2015a: 377).

I3 Il romanzo contiene altri passi delle lettere. La fonte è segnalata da Pennacchi 20I5a: 428 nella Bibliografia essenziale: «L'epistolario Claretta-Benito è in L. Montevecchi (a cura di), Benito Mussolini. A Clara. Tutte le lettere a Clara Petacci 1943-1945, Milano 201I».

I4 Lo riporta anche <http://www.carabinieri.it/arma/curiosita/non-tutti-sanno-che/z/luigi-fiorello-marziale-zaccheo $>$. Alla sua memoria (precisa il sito) è intitolata, dal 5 settembre 
I929 [...] scrisse : "Oggi qualcuno crederà di riaprire le porte di secoli in cui s'intrecciarono lo scettro e il pastorale. Ma la realtà del XX secolo non tarderà a farsi sentire, le grandi masse ricompariranno dietro allo scenario"», Pennacchi 20I5a: $\left.2 \mathrm{IO}^{15}\right)$ e per il ricordo che di lui lasciò Togliatti nel $\mathrm{I} 955 \mathrm{su}$ «Rinascita» ${ }^{16}$.

Questo non significa naturalmente che l'italiano tocchi solo la sfera scritta: asistematico - lo si è già detto - è il procedimento di Pennacchi, che alterna (con effetto di naturalezza) dialetto e italiano. Il narratore stesso tende a un italiano medio-basso, non privo di inflessioni, che tuttavia - nel riportare più efficacemente un pensiero - talvolta non rinuncia al dialetto ${ }^{17}$. L'italiano può inoltre entrare in campo in scene in cui a parlare sono personaggi la cui reale esistenza è ambigua (in italiano si esprime la misteriosa figura femminile che, secondo zia Pace e di zio Adelchi, è il fantasma di Claretta Petacci $\left.{ }^{18}\right)$; in momenti in cui ad esprimersi è una collettività indefinita ${ }^{19}$; oppure in situazioni che implicano la necessità di nascondersi dietro a una parlata neutra, per celare la sfera delle emozioni. Particolarmente significativi in questo senso sono due passi situati alla fine del romanzo: il dialogo fra Diomede e zio Adelchi e l'incontro onirico-fantastico fra Diomede e Eberhard.

2007, la Caserma sede della Compagnia Carabinieri di Aprilia (LT).

I5 Anche in questo caso il documento è autentico: vd. De Gasperi 1974. La lettera è alle pp. 78-8o del vol. I.

I6 «Il povero De Gasperi morirà infatti nel 1954 e Togliatti, ricordandolo l'anno dopo su "Rinascita", scriverà perfino che le poche riforme strutturali della politica centrista - soprattutto la riforma agraria e l'istituzione della Cassa per il Mezzogiorno - erano state fortemente volute non tanto da De Gasperi quanto dalla sinistra democristiana, andando lui invece ricordato come l'oggettivo restitutore del "potere economico a una classe dirigente capitalistica chiusa, egoistica, che non ha prospettive davanti a sé"» (Pennacchi 2015: 211). Il passo è ora disponibile in Togliatti 2014.

I7 Si veda l'esempio seguente: «È l'antropologia. L'uomo è fatto così, ha un bisogno vitale di relazionarsi con gli altri, ha bisogno - per essere felice - di sentirsi amato. E il modo più semplice di farci amare è camminare insieme agli altri, andare dove vanno gli altri, dire quello che dicono gli altri. Se vai in un'altra direzione il flusso ti travolge, fai più fatica a muoverti, sei un diverso. "Casso sìto drìo fare?" ti dicono: "Non lo vedi che intralci?"» (2015a: 153).

I8 «Sparita dissolta nel nulla, nel buio della notte. "Porca putana!" aveva fatto zio Adelchi, con la mano - bisogna dire - subito alla pistola.| "Fàntasimi" disse allora zia Pace a Diomede, "e tanta altra gente l'agà vista". |"Ma qua' spìriti fantàsimi, zia?" ridisse piangendo mio cugino Diomede di appena diciannove anni: "E questo cossa xèo, alora?" tirando fuori dalla tasca l'orecchino d'oro bianco e i brillantini con la perla incastonata, tale e quale a quello della foto: "Xèo un fantasma anca questo?"»(Pennacchi 2015a: 318).

I9 «C'è pure chi dice che la resistenza in Italia non ci sarebbe mai stata: "È un mito inventato a posteriori per legittimare la repubblica democratica antifascista. Non ci fu nessun movimento di resistenza attiva, nessuna lotta di popolo. I cosiddetti partigiani non furono, in realtà che pochi delinquenti assassini disertori”»(Pennacchi 2015a: I86). 
Nel primo esempio - ormai con una posizione lavorativa e sociale consolidata - il grande protagonista di questa Seconda parte inizialmente tenta di reagire con oggettività ferma e determinata alle richieste dello zio (esprimendosi in italiano):

\begin{abstract}
Ah, no no no no, zio! Noi siamo Peruzzi, siamo gente per bene, pionieri bonificatori, queste cose non le facciamo, non vado a sporcare il buon nome della famiglia per una cosa così riprovevole, corruzione, concussione, favoreggiamento, clientelismo, paternalismo, parentopoli, raccomandazioni. Mi meraviglio di voi, zio, che siete sempre stato un esempio adamantino, venirmi adesso a chiedere una cosa così... (2015a: 4I2).
\end{abstract}

Tuttavia l'insistenza e le provocazioni dello zio gradualmente accendono in Diomede l'ira rabbiosa e, di conseguenza, la scintilla del dialetto che culmina nel «Fora de qua, asasin! Bruto vecio sporco maledeto fiòl d'un can, asasin de nevodi e de la brava zente» (2015a: 413).

La seconda scena presenta un altro, più fragile, volto di Big Boss. La conquista sociale e la conquista linguistica si affiancano a una graduale perdita: perdita della memoria dei nomi (chiama la moglie «cara» perché come si chiami, a tratti non lo ricorda) e dei luoghi; e nel finale, perdendosi (“'Siete del posto?", ha chiesto: "Che città è?”», 2015a: 423), si ritrova: ritrova l'amico Eberhard e il dialetto, in un'ambientazione di onirico conforto, che si conclude con quella sorta di frase-ritornello che accompagnava i lunghi tempi trascorsi insieme in gioventù ("Vorìa star senpre qua" diceva Diomede»). Ma questa volta non gli fa eco la consueta e passata risposta dell'amico («Anca mi" rispondeva il tedesco» [2015a: 29]):

Poi ha sentito delle carriole che facevano "Cìo-cìo» e all'improvviso è spuntato Eberhard vicino a lui, l'amico suo tedesco. | Ed Eberhard gli ha sorriso. Lentamente ha sbollato ed aperto un pacchetto nuovo di sigarette. Ha estratto la prima e gliel'ha offerta. | «Fumemo, va', gli ha detto. | Diomede s'è rilassato. E mentre il cigolare di carriole - «Cìo-cio-ciìoooo...» - lentamente s'allontanava e poi cessava, hanno fumato seduti a fianco sul muretto, con le spalle abbandonate alla ringhiera. | «Ah, vorìa star senpre qua...» ha sospirato Diomede (2015a: 424).

Diomede si perde nei luoghi di un'intera vita, ma, perdendosi, ritrova nella memoria più intima la sua lingua (e sempre lì vorrebbe stare) che ora, e solo ora, nell'ideale riunione, può nuovamente prestare a Eberhard ( $(\mathrm{Fu}$ memo, va» 2015a: 424). Così si chiude la Parte seconda di Canale Mussolini: su un disorientamento spaziale ed esistenziale. E sul tempo in cui «a Latina, cominciarono a chiudere le prime fabbriche» (2015a: 425): siamo all'inizio degli anni Sessanta e verso Nord si spostano zio Temistocle e i suoi figli («i 
figli lo riportarono in Altitalia negli anni Sessanta, a Torino. Loro andavano in fabbrica, alla Fiat, e lui li aspettava a casa», 20IO: I2).

\section{Verso Nord: note conclusive}

Se l'«esodo» verso Sud accomuna migliaia di persone che lavorano per la bonifica, negli anni del boom economico, lo spostamento è di segno opposto ed è più ramificato poiché coinvolge anche lo spostamento dalla campagna alla città, in particolare nel cosiddetto «triangolo industriale», costituito da Milano, Genova e Torino. La questione non è semplicemente economica, ma è anche urbanistica e sociale, dal momento che il fenomeno del «miracolo economico», rappresenta

[...] l'occasione per un rimescolamento senza precedenti della popolazione italiana. Centinaia di migliaia di italiani [...] [partono] dai luoghi d'origine, [lasciano] i paesi dove le loro famiglie avevano vissuto per generazioni, [abbandonano] il mondo immutabile dell'Italia contadina e [iniziano] nuove vite nelle dinamiche città dell'Italia industrializzata (Ginsborg I989: 294).

Tutto ciò, naturalmente, va collocato anche entro il dibattito attento al rapporto fra letteratura e industria, che trova tra l'altro ampio spazio, come è noto, nel IV fascicolo (del I96I) de «Il Menabò»: della tematica basti qui ricordare che la sua essenza ideologica vede (come appare evidente nell'intervento di Vittorini Industria e letteratura) nel «rinnovamento del linguaggio la possibilità di una letteratura adeguata ai temi umani più scottanti della società neocapitalista» (Manacorda 1996: 448).

Ma come vivono, come lavorano e, soprattutto, come parlano i personaggi che sono al centro di questa nuova realtà? Pennacchi non lo racconta; lo raccontano, però, fra gli altri - in presa diretta, senza alcuno scarto temporale fra vicenda narrata e atto della scrittura - il protagonista de La vita agra (1962) di Bianciardi che dalla provincia toscana si sposta a Milano e l'operaio Albino Saluggia del Memoriale (1962) di Volponi. Questi testi - cui andrebbe aggiunta Una nuvola dira di Arpino (anch'esso del 1962) - attirano l'attenzione di un lettore attento quale Italo Calvino che quello stesso anno, ne La tematica industriale (uscita su «Il Menabò», n. 5, I962), si sofferma sulla loro lingua: a proposito di Bianciardi scrive che «[l]'assunto linguistico [...] dimostra di poter servire a rappresentare ed esprimere un quadro e un giudizio della realtà industriale più complesso [...], anche se qui non si esce dai limiti di una protesta anarchico-privata» (Calvino 1962: 34-35); quanto a Volponi osserva che «l'impostazione linguistica di Volponi è la più inattesa, 
data la "tematica industriale", eppure è quella che dà il risultato poetico più alto» $\left(\right.$ I962-35) ${ }^{20}$.

Le riflessioni di Calvino indicano, nei due romanzi, una particolare attenzione per la lingua, che tuttavia non si materializza nell'uso del dialetto e nemmeno in un impiego imponente di una parlata regionale. Se le scelte linguistiche costituiscono un tratto importante (anche) di questi romanzi, i diversi esiti dipendono forse dal venir meno della necessità di dare voce a una collettività, a favore della voce del singolo. Un singolo che - naturalmente - può anche essere il rappresentante di una società, ma che singolo resta, poiché quella società non concepisce l'idea di una comunità: è la solitudine a dominare. Pertanto una tale attenzione è segno di sensibilità per un cambiamento in atto nella società, vale a dire la disgregazione e il disorientamento cui il nuovo cittadino, immerso in un paese che fatica a riconoscere, è confrontato. In questa esistenza alienata anche il dialogo con l'altro (con gli altri) si affievolisce. E in effetti - oltre ad essere accomunati da un contatto violento con la nuova realtà ${ }^{21}-\mathrm{i}$ protagonisti dei romanzi di Bianciardi e Volponi sono accomunati dalla solitudine più assoluta.

L'isolamento di fronte al quale viene a trovarsi il protagonista de La vita agra è, in fondo, presentato con estrema lucidità anche nel romanzo precedente di Bianciardi (L’integrazione [1960]), ove l'individuo si trova di fronte a una città-cantiere:

[...] la città, come era scritto nei giornali, era veramente tutta un cantiere. Dinnanzi alla facciata chiusa e scura delle case, operai febbrili alzavano tavolati [...]. Dietro il tavolato fervevano lavori. Nella maggior parte dei casi il lavoro consisteva nel buttare giù una casa. Poi costruivano; più alto e più brutto (Bianciardi I960: I6-I7).

E in questo cantiere cittadino tutti «corrono come allucinati dalla mattina alla sera, per comprarsi quello che credono di desiderare; in realtà quel che al padrone piace che si desideri» (1960: 31); chi rimane indietro - chi fatica a integrarsi - rimane solo («Così noi due restavamo soli soli per strada, con dodici ore disponibili di fronte a noi, prima che cominciasse un altro

20 Più severo il giudizio su Arpino: «Arpino (come molti altri prima di lui) ha voluto far parlare (e pensare) i suoi operai con un linguaggio unitario che insieme fosse parlato-popolare ed esprimesse una coscienza etico-politico-culturale. Già la letteratura impegnata del dopoguerra [...] aveva cercato d'introdurre nei romanzi questo parlar di politica in termini popolar-colloquiali e poetico-allusivi, quasi cercando di saltare a pié pari il fosso che separa linguaggio ideologico, linguaggio quotidiano linguaggio letterario. Questa sintesi non è avvenuta; e ora Arpino inciampa nello stesso fosso» (Calvino 1962:34).

2I Per Albino Saluggia si tratta di un contatto diretto con la fabbrica e con il mondo operaio; per il protagonista de La vita agra, il contatto con la fabbrica non ci sarà, ma violentissima sarà l'immersione nella nuova vita (non-vita) prodotta dal mondo industriale. 
giorno», I960: 25). Solo rimane anche l'intellettuale-traduttore de La vita agra, che a Milano giunge spinto soprattutto da un desiderio di vendetta, una vendetta mai consumata, poiché il meccanismo perverso della città lo costringe a una vita da automa, che gli fa perfino dimenticare la sua missione. La sua esistenza è costellata da pochi incontri significativi, poiché a dominare sono figure anonime, «larve $»^{22}$. Alla persona si sostituisce l'immagine:

E poi mi sono accorto che andando in centro trovi sì qualche conoscenza, ma ti accorgi subito che la tua conoscenza è un fatto puramente ottico. Non trovi le persone, ma soltanto la loro immagine, il loro spettro, trovi i baccelloni, gli ultracorpi, gli ectoplasmi. Nei primi mesi del loro arrivo in città forse no, forse resistono e hanno ancora una consistenza fisica, ma basta un mezzo anno perché si vuotino di dentro, perdano linfa e sangue, diventino gusci. Scivolano sul marciapiede rapide e senza rumore, si fermano appena al saluto, con un sorriso scialbo (e anche all'esterno, se guardi bene, sono già un poco diversi, cioè impinguati e sbiancati) (Bianciardi 1962: 194).

In questo clima la lingua de La vita agra $^{23}$ ricalca la rabbia di chi non accetta il sistema, non si integra (rimanendo ancorato alla terra d'origine) e vive nel presente (senza scarto fra atto della scrittura ed esperienza vissuta). La rabbia implica una «una mescidazione di codici (talora persino un code mixing in senso proprio)» (Zublena 2007: $47^{24}$ ) su una base di «italiano medio agile» (2007: 54). Entro tale quadro i dialetti presenti sono essenzialmente due: il «toscano dell'autore-narratore» e il «milanese d'ambientazione» (2007: 55). Diversamente da quanto accade in Pennacchi, ove il dialetto «straniero» si piega al veneto, in Bianciardi - che scrive nel momento in cui vive quell'esperienza - il milanese mira a enfatizzare il disorientamento del protagonista, che si registra sia nella quotidianità (“Come va?” gli chiesi. "Vuoi una mano?" | Brontolò qualcosa in dialetto, di gola, si tirò su a sedere

22 «Spesso la sera, dopo rigovernati i piatti, uscivamo a passeggio nella nebbia. Fuori non s'incontrava una persona, soltanto nel cono di luce sporca dei lampioni qualche larva imbacuccata e frettolosa [...]» (1962: 98); «Abbandonata, la terra aveva buttato su sterpi, erbacce [...]. Di giorno ci andavano i ragazzini a giocare agli indiani, ma di notte si riempiva di larve indistinte in quella scarsa luce frammezzo alla nebbia che si abbioccolava sugli sterpi. | [...] | Era una bolgia di purgatorio, e mai ho saputo con precisione se quella larve fossero uomini oppure donne, persone vere o fantasmi» (I962: IOO-OI).

23 Sulla lingua de La vita agra fondamentali sono i saggi di Grignani 1992 e Zublena 2007, cui si rimanda per uno studio completo e approfondito.

24 Più precisamente Zublena parla di «una mescidazione di codici [...] fatta dalla compresenza di dialettismi lombardi (ma non solo), toscanismi, colloquialismi (lessicali, morfosintattici e sintattici), forestierismi, tecnicismi, gergalismi, neologismi d'autore, il tutto condito da un forte grado di intertestualità che richiede un'enciclopedia piuttosto vasta [...]» (Zublena 2007: 47). 
e mi tese la mano. Avevo capito che intendeva dirmi aiutami a rialzarmi in piedi [...]», Bianciardi I962: I03), sia al lavoro («Locuzioni dialettali. Lei ha questo difetto, le locuzioni dialettali, come tutti i toscani, del resto [...]», I962: I28), sia in un'ideale forma di comunicazione politica («Succede che qualcuno metta il piede in fallo e finisca sotto le ruote. [...] "L'era il Gino", informa uno, nel silenzio. Io mi chiedevo se ci fosse modo di conoscerli, questi compagni di Tacconi Otello, di parlarci, superando la difficoltà dei dialetti [...]», 1962: 56). Lo scoglio del dialetto si traduce dunque in impossibilità di dialogo - attraverso situazioni in cui «l'effetto comico si accompagna a una forte componente di critica sociale» (Zublena 2007: 57) 25 $^{25}$ e l'unica forma di difesa sembra essere la fedeltà al toscano che, pur impiegato «come macchia di colore quasi folkloristica» quando coinvolge «porzioni di testo superiori al singolo lessema o sintagma» $(2007: 55)^{26}$, si trasforma in "vettore di identità» quando a essere coinvolto è un lessema «singolo, inserito in un contesto di italiano comune» (2007: 60 $)^{27}$. Viceversa Pennacchi - nel suo impiego dilagante del veneto-pontino - sottolinea un'epopea trasmessa oralmente (quasi «dettata» dalle generazioni precedenti).

Ancora più alienante la solitudine di Albino Saluggia («[D]i nuovo solo ed escluso come ai tempi di Vattimo e della prigionia», Volponi I962: 72), che si muove fra campagna e città-fabbrica. Loperaio al centro di Memoriale, nella sua nevrosi, sembra trovare una forma di colloquio appagante non con le persone, ma con la fabbrica:

Amavo a poco a poco la fabbrica, sempre di più man mano che m'interessava meno la gente che vi lavorava. Mi sembrava infatti che tutti gli operai avessero poco a che fare con la fabbrica, che fossero o degli abusivi o dei nemici, che non si rendessero conto della sua sovrumana bellezza e che proprio per questo, lavorando con più fracasso del necessario, parlando e ridendo, la offendessero deliberatamente. [...] La fabbrica mi appariva sempre più bella $\mathrm{e}$ mi sembrava che si rivolgesse direttamente a me, come se fossi l'unico o uno dei pochi in grado e ben disposto a capirla (1962: 50).

25 Si leggano i due esempi seguenti: «Dicevano tutti la grana. La grana e poi i dané. La grana sarebbe quella che si prende, i dané quelli che si pagano, mi pare di aver capito» (Bianciardi I962: IOI). E, poco oltre, si veda la scena degli acquisti in drogheria: «"E poi?"| "Una bottiglia di acquetta."| "Cosa l'è l'acquetta?"| "La varechina." |"Sa l'è la varechina?" | "La candeggina." | "Ah, e poi?"» (I962: IOI-O2).

26 Si veda l'esempio citato da Zublena 2007: 55: «Nciavresti mia nposticino da guadagnà bbene senza lavorà tanto? Sai omè, sulle cencinquanta rmese? Giù, madonnarbuio, un si batte iodo. Un si trova nalira peffacantà nceo» (1962:32).

27 Ancora una volta il rinvio - per la ricca serie di esempi e le spiegazioni - è a Zublena 1997 : 6I-62 e, prima ancora, a Grignani 1992: 56. 
Nel presentare un personaggio che rimuove la possibilità del dialogo con l'altro, crea un rapporto di esclusività con la fabbrica ${ }^{28}$, quasi fosse stato scelto da lei come interlocutore unico («mi sembrava che si rivolgesse direttamente a me»), e conduce un'esistenza di solitaria prigionia (enfatizzata nel romanzo dalla presenza ossessiva della prima persona ${ }^{29}$ ), Volponi opta - linguisticamente - per una scelta molto lontana da quella di Bianciardi. Assolutamente estraneo al «pastiche dialettale» (Zinato 200I: I26), l'autore di Memoriale non ricalca la lingua di un operaio, non cerca di individuarne le possibili inflessioni linguistiche, ma lo «salva» demolendo la realtà della prigionia di Albino, «formando un unico vetro dipinto su due strati» (Pasolini 1962: 209), in cui alla "poeticità naturale del grafomane appartenente alla classe inferiore che usa una lingua non sua, appresa nei libri letti in ospedale o in prigione» (1962: 209), si sovrappone «la sua poeticità personale - anch'essa a suo modo personale - di poeta colto che ha letto i migliori poeti del Novecento fino a Bertolucci e dopo» (1962: 209). Questo significa che l'unica forma di rappresentazione dell'essenza di Albino Saluggia, agli occhi di Volponi, non può essere né il dialetto né la parlata regionale:

\begin{abstract}
Nel freddo di gennaio, di un gennaio senza neve ma molto freddo, ero all'aperto e solo, minacciato di morte. Due scavatrici lavoravano ai lati del terreno con grande rumore; ruotavano e di dirigevano l'una contro l'altra. Si fermavano, facevano un altro giro e riprendevano a muoversi in senso contrario. Guardavo questo lavoro seduto su un attrezzo, all'orlo della strada (Volponi 1962: 79).
\end{abstract}

La lingua di Volponi traduce lo sguardo poetico e alienato (e doloroso nella sua ingenuità), di chi non è in grado di affrontare un cambiamento economico, sociale e culturale straordinario. Anche Bianciardi - attraverso scelte diverse - cerca la coerenza fra la persona (non il «guscio»!) e l'espressione linguistica. In entrambi i casi - assolutamente in Volponi, parzialmen-

28 Sette anni dopo la pubblicazione di Memoriale, nel 1969, uscirà Il crematorio di Vienna di Goffredo Parise. Anch'esso traccia (ma con una durissima critica sociale) il delinearsi dell'individuo sempre più alienato. Si veda, a titolo d'esempio, il passo seguente (dal racconto Io): «Non è raro che mi soffermi a contemplare una casa, oppure un'automobile, che non è nemmeno di mia proprietà. E certe volte mi sorprendo a intavolare una conversazione occasionale appunto con un'automobile o con certi oggetti che sono esposti nelle vetrina. [...] imbastisco la conversazione da solo, cioè come se parlassi da solo, mentre in realtà io comunico con loro molto più che con i miei conoscenti, con mia moglie e i miei figli» (Parise 1969: 79).

29 Di seguito una serie di esempi: «Battevo i miei passi anch'io come uno scolaro» (Volponi I962: 57); "Andai camminando intorno alla fabbrica ed arrivai, dopo tutte le costruzioni industriali, verso la campagna» (79); «Io guardavo i passeri e pensavo che era incredibile che fossero così vicino alla cattiveria umana» (83); «Come sempre, mi consolai camminando per la strada verso casa mia» (II3); «Uscito dalla fabbrica andavo adagio verso il centro della città» (I32); «Andai al cinema a Torino e trovai la città disabitata» (I5I); «[...] e scesi per la città dalla parte opposta della fabbrica, verso il fiume, tra le ombre degli orti» (I58). 
te in Bianciardi - il dialetto non costituisce una spinta (o una forza) aggregante, forse poiché al centro vi è un individuo, non una collettività. Quella collettività che invece è fondamentale nella prima e nella seconda parte di Canale Mussolini («Quel romanzo parla della storia di tutti. È la voce collettiva. Io l'ho solo messa in pagina e fatta cantare», Pennacchi 20II).

E forse, a questo punto, non è un caso se il dialetto domina invece in un'altra opera dell'inizio degli anni Sessanta, vale a dire Il calzolaio di Vigevano di Lucio Mastronardi (edito in volume nel 1962, ma uscito già nel I959), appartenente a un progetto più ampio (costituito da Il maestro di Vigevano e Il meridionale di Vigevano $^{30}$ ): una trilogia che - analogamente a quanto avviene in Pennacchi - traccia l'incontro fra la grande Storia e la storia di una cittadina attraverso una famiglia ${ }^{31}$. Un'altra saga famigliare; e un'altra saga in cui fra atto della scrittura e fatti narrati vi è uno scarto temporale ${ }^{32}$. Spazio e tempo, dunque, ma anche - dando voce a Mastronardi, in un'intervista del I975 - «novità delle parole»: «Vorrei poter descrivere la vita in pochi secondi. Anche le novità delle parole, del loro uso, l'uso dei verbi, fanno parte di una ricerca [...] che riguarda il tempo e lo spazio» (Mastronardi 1975: XVI) ${ }^{33}$.

\section{Bibliografia}

Belloni, Silviano, Grammatica veneta, Padova, Esedra 2009.

Bianciardi, Luciano, L’integrazione [I960], Milano, Feltrinelli, 2014.

—. La vita agra [1962], Milano, Feltrinelli, Milano, Feltrinelli 2013.

Calvino, Italo, La tematica industriale, «Il Menabò» (pp. I8-2I), n. 5, I962, ora in Id., Mondo scritto e mondo parlato, Milano, Mondadori, 2002 (da cui si cita).

- Ricordo di Lucio Mastronardi, in Per Mastronardi. Atti del Convegno di studi su Lucio Mastronardi, Vigevano 6-7 giugno I98I, a cura di Maria Antonietta Grignani, Firenze, La Nuova Italia, I983, ora in Mastronardi, Lucio, Il maestro di Vigevano. Il calzolaio di Vigevano. Il meridionale di Vigevano, cit., pp. 447-449.

30 Il calzolaio di Vigevano appare sul «Menabò» del 1959 e viene quindi pubblicato da Einaudi nel 1962 (e presso Einaudi, nello stesso anno, usciva Il maestro di Vigevano), mentre nel 1964 vede la luce (ancora per Einaudi) Il meridionale di Vigevano.

3I Ancora secondo Calvino il valore del Calzolaio in quanto «romanzo storico» «sta [...] nel rendere come nessun altro la continuità italiana tra fascismo e post-fascismo, e questo in un'epoca in cui ancora il tema storico della nostra narrativa era la separazione dal presente di quel passato» (Calvino 198I: 448).

32 «[...] se è vero», scrive Calvino, «che Il calzolaio si svolge tra la guerra d'Abissinia e l'inizio del dopoguerra, la carica propulsiva gli viene certo dagli anni in cui fu scritto che erano i primi anni del boom» (Calvino I98I: 448).

33 Le parole dell'intervista si trovano nell'Introduzione di Maria Antonietta Grignani a Mastronardi 1962. 
De Gasperi, Maria Romana (a cura di), De Gasperi scrive. Corrispondenza con capi di Stato, cardinali, uomini politici, giornalisti, diplomatici, Brescia, Morcelliana, I974.

Ginsborg, Paul, Storia d'Italia dal dopoguerra ad oggi, Torino, Einaudi, I989. Ginzburg, Natalia, Lessico famigliare, Torino, Einaudi, 1963.

Grignani, Maria Antonietta, La lingua 'agra' di Luciano Bianciardi, in Luciano Bianciardi tra neocapitalismo e contestazione, a cura di Velio Albati et al., Roma, editori Riuniti, I992, pp. 89-I07, ora in Id., Novecento plurale. Scrittori e lingua, Napoli, Liguori, 2007, pp. 49-67 (da cui si cita).

Manacorda, Giuliano, Storia della letteratura italiana contemporanea (I94OI996), Roma, editori Riuniti, I996.

Montevecchi, Luisa (a cura di), Benito Mussolini. A Clara. Tutte le lettere a Clara Petacci 1943-1945, Milano, Mondadori, 201 I.

Mastronardi, Lucio, Il calzolaio di Vigevano [1959/1962], in Id. Il maestro di Vigevano. Il calzolaio di Vigevano. Il meridionale di Vigevano, Introduzione di Maria Antonietta Grignani, in appendice scritti di Italo Calvino e Gian Carlo Ferretti, Glossario di Valentina Zerbi, Torino, Einaudi, 2016.

-. Il maestro di Vigevano [1962], in Id., Il maestro di Vigevano. Il calzolaio di Vigevano. Il meridionale di Vigevano, cit.

-. Il meridionale di Vigevano [I964], Il maestro di Vigevano. Il calzolaio di Vigevano. Il meridionale di Vigevano, cit.

Nardo, Luigi, Dizionario italiano-veneto. A sercar parole, Padova, Editoriale Programma, 2009.

Pasolini, Pier Paolo, Il mostro e la fabbrica, "Paese sera», I3.04.1962, e ora in Zinato, Emanuele, Volponi, cit., pp. 209-2I2 (da cui si cita).

Parise, Goffredo, Il crematorio di Vienna [I969], Introduzione di Silvio Perella, Milano, Mondadori, I993.

Pennacchi, Antonio, Canale Mussolini [2010], Milano, Mondadori, 2015.

- Scrivere non mi diverte volevo fare il pugile [intervista di Antonio Gnoli], «la Repubblica», 2I.0I.20II.

-. Canale Mussolini. Parte seconda [2015], Milano, Mondadori, 2015 [20I5a].

- La fatica di scrivere: Antonio Pennacchi e la seconda parte di «Canale Mussolini» [intervista di Matteo Nucci], in <http://www.minimaetmoralia.it/ wp/la-fatica-di-scrivere-antonio-pennacchi-e-la-seconda-parte-di-canale-mussolini/>, 08.12.20I5 [2015b].

Testa, Enrico, Lo stile semplice. Discorso e romanzo, Torino, Einaudi, 1997.

Togliatti, Palmiro, La politica nel pensiero e nell'azione: Scritti e discorsi 19I7I964, a cura di Michele Ciliberto e Giuseppe Vacca, Milano, Bompiani, 2014.

Vittorini, Elio, Industria e letteratura, «Il Menabò», n. 4, I96I.

Volponi, Paolo, Memoriale [1962], Torino, Einaudi, 2015.

Zinato, Emanuele, Volponi, Firenze-Palermo, Palumbo, 200 . 
GeOrgia Fioroni

Zublena, Paolo, Dentro e fuori la scrittura anarchica. La Lingua della «Vita agra» di Bianciardi, «il Verri» (vol. 52, edizione 38), 2007. 